\title{
Modélisation mathématique de la transmission de la COVID-19 et stratégies d'atténuation des risques dans la population ontarienne au Canada
}

\author{
Ashleigh R. Tuite PhD MSP, David N. Fisman MD MSP, Amy L. Greer PhD MSc
}

Citation : CMAJ 2020 May 11;192:E497-505. doi : 10.1503/cmaj.200476-f; diffusion hâtive le 8 avril 2020

Voir la version anglaise de l'article ici : www.cmaj.ca/lookup/doi/10.1503/cmaj.200476; voir l'éditorial connexe (en anglais et français) ici : www.cmaj.ca/lookup/doi/10.1503/cmaj.200606

\section{RÉSUMÉ}

CONTEXTE : Au Canada, on utilise des interventions d'éloignement physique pour ralentir la propagation du SRASCoV-2 (coronavirus du syndrome respiratoire aigu sévère 2 ), mais on ignore au juste quelle en sera l'efficacité. Nous avons évalué comment différentes interventions non pharmacologiques pouvaient être utilisées pour maîtriser la pandémie de COVID-19 (maladie à coronavirus 2019) et alléger le fardeau qu'elle impose au système de santé.

MÉTHODES : Nous avons utilisé un modèle à compartiments structuré selon l'âge pour faire une analyse de la transmission de la COVID-19 dans la population de l'Ontario, au Canada. Nous avons comparé un scénario de référence, soit dépistage limité, isolement et quarantaine, à des scénarios incluant dépistage accru, mesures strictes d'éloignement physique, ou combinaison de dépistage accru et d'éloignement physique moins strict. Les interventions étaient appliquées soit pendant des durées fixes, soit selon un cycle dynamique en fonction de l'occupation projetée des lits dans les unités de soins intensifs (USI). Nous présentons les médianes et les intervalles de crédibilité tirés de 100 expériences répliquées par scénario sur un horizon temporel de 2 ans.

RÉSULTATS : Selon le scénario de référence, nous avons estimé que $56 \%$ (intervalle de crédibilité de 95\%, 42\%$63 \%$ ) de la population ontarienne contractait l'infection pendant l'épidémie. Au moment du sommet épidémique, nous avons projeté 107000 (intervalle de crédibilité de 95\%, 60760-149000) hospitalisations (soins standards) et 55500 (intervalle de crédibilité de 95\%, 32700$75200)$ hospitalisations dans les USI. Pour les scénarios à durée fixe, selon les projections, toutes les interventions retardaient et réduisaient la hauteur du sommet épidémique par rapport au scénario de référence, et ce sont les mesures d'éloignement physique strict qui exerçaient le plus d'effet; de même, les interventions de durée plus longue étaient plus efficaces. Selon les projections, les interventions dynamiques réduisaient la proportion de la population atteinte à la fin de la période de 2 ans et pouvaient ramener le nombre médian de cas dans les USI en deçà des estimations actuelles de leur capacité en Ontario.

INTERPRÉTATION : Sans éloignement physique substantiel ou une combinaison d'éloignement physique modéré et de dépistage accru, nous projetons que les ressources des USI pourraient être insuffisantes. L'éloignement physique dynamique maintiendrait la capacité du système de santé en plus de donner un répit psychologique et économique périodique aux populations.
$\mathbf{L}$

a pandémie de COVID-19 (maladie à coronavirus 2019) représente une urgence mondiale de santé publique sans précédent dans l'histoire récente. Depuis le rapport initial de l'Organisation mondiale de la santé il y a 2 mois qui décrivait l'épidémie de COVID-19 concentrée à Wuhan, en Chine ${ }^{1}$, le nombre de cas confirmés est brutalement passé de 282 à plus de 330000 , et 14510 décès ont été signalés dans toutes les régions du monde 2 . Le premier cas importé de COVID-19 en Ontario, au
Canada, a été rapporté le 25 janvier 2020, et la transmission communautaire a été documentée pour la première fois le $1^{\mathrm{er}}$ mars 2020, en Colombie-Britannique, au Canada ${ }^{3}$.

Cet agent pathogène représente un défi de taille pour la santé publique, la planification de la lutte à la pandémie et les systèmes de santé. Le coronavirus du syndrome respiratoire aigu sévère 2 (SRAS-CoV-2) est hautement transmissible ${ }^{4-7}$. Il provoque des symptômes cliniques de modérés à graves chez 
environ $20 \%$ de tous les individus atteints diagnostiqués ${ }^{5,8,9}$. En l'absence d'un vaccin, la réponse de la santé publique s'est concentrée sur l'utilisation d'interventions non pharmacologiques ${ }^{10}$, qui incluent des mesures liées au scénario de référence, telles que dépistage, recherche des contacts, isolement des cas atteints et mise en quarantaine des cas exposés, et des mesures non liées au scénario de référence, telles que réduction de la probabilité de transmission par contact réel (lavage des mains et hygiène respiratoire) et éloignement physique, afin de réduire le taux de contacts dans la population. Les mesures d'éloignement physique réduisent les possibilités de transmission du virus d'une personne à l'autre. Elles incluent, ensemble ou séparément, la fermeture des écoles, le télétravail, l'annulation des activités et événements de groupes et une réduction généralisée des contacts dans la communauté. Même si on s'attend à ce que ces mesures réduisent efficacement la transmission du SRASCoV-2, elles ont un coût substantiel et perturbent la vie sociale.

Les modèles épidémiologiques peuvent fournir des pistes importantes aux gestionnaires de la santé publique en permettant l'examen de divers scénarios hypothétiques. Le Plan canadien de lutte contre la pandémie d'influenza pour le secteur de la santé, dont la structure guide la préparation et la réponse à la pandémie de COVID-19, détermine 2 principaux objectifs de lutte à la pandémie : réduire la morbidité grave et la mortalité, et prévenir les bouleversements sociaux ${ }^{11}$. L'objectif primordial d'une réponse à la pandémie est de trouver une combinaison d'interventions non pharmacologiques aptes à réduire le nombre d'hospitalisations (soins standards et soins intensifs) et de décès, tout en limitant la perturbation sociale. Il serait possible de limiter la perturbation sociale en abrégeant dans l'ensemble l'application des interventions qui visent à réduire la morbidité et la mortalité. L'un des défis de la réponse à la pandémie est que, dans une population qui est susceptible d'être atteinte de la maladie, même si, pendant leur application, les interventions non pharmacologiques en ralentissent la transmission, une fois levées (ou lorsque le respect des consignes se relâche), la transmission de l'agent pathogène connaît rapidement un rebond $d^{10,12}$. Dans le cas de la COVID-19, il pourrait être impossible de réduire simultanément la morbidité/mortalité et la perturbation sociale et économique.

C'est pourquoi nous avons utilisé un modèle dynamique de transmission de la COVID-19 pour explorer les répercussions potentielles des interventions non pharmacologiques, liées ou non au scénario de référence, sur la population de l'Ontario, au Canada. Notre analyse se concentre sur la définition de stratégies visant à maintenir le nombre de cas graves projeté (soins standards et soins intensifs) dans une fourchette qui ne surchargera pas le système de santé ontarien, en tenant compte de leur durée d'application.

\section{Méthodes}

\section{Aperçu du modèle}

Nous avons conçu un modèle à compartiments structuré selon l'âge qui décrit la transmission de la COVID-19 dans la province de l'Ontario, au Canada. Nous avons utilisé un cadre SEIR (sus- ceptible, exposé, infectieux, rétabli) modifié par l'ajout de compartiments additionnels pour tenir compte des interventions de la santé publique, de l'intensité variable des symptômes cliniques et du risque d'hospitalisation. Un aperçu des compartiments du modèle et de leurs interactions est fourni à la figure 1; les équations et détails additionnels du modèle sont fournis à l'annexe 1, accessible (en anglais) ici : www.cmaj.ca/lookup/ suppl/doi:10.1503/cmaj.200476/-/DC1. Le modèle a été échelonné sur une période de 2 ans, et nous avons présumé que les individus rétablis restaient immunisés contre une réinfection pendant la durée de l'épidémie. Les individus demeuraient infectieux jusqu'à leur rétablissement ou leur hospitalisation; nous n'avons pas modélisé la transmission dans les milieux de soins. Pour simplifier, nous avons présumé que tous les décès concernaient les cas de soins intensifs. Nous avons inclus les cas sous soins standards et sous soins intensifs pour estimer les besoins médicaux pour la période de l'épidémie. Le modèle a été élaboré avec le logiciel $\mathrm{R}^{13}$.

\section{Paramètres du modèle}

Le modèle a été stratifié par tranches d'âge de 5 ans à partir des estimations de population de $2019^{14}$. Les contacts à l'intérieur et entre les tranches d'âge se fondent sur l'étude POLYMOD ${ }^{15}$, sur les données de contact spécifiques au Royaume-Uni. Le modèle a ensuite été stratifié par état de santé pour tenir compte de la vulnérabilité différentielle à l'infection sévère chez les individus ayant des problèmes de santé sous-jacents. Nous avons obtenu les estimations de comorbidités selon l'âge à partir de l'Enquête sur la santé dans les collectivités canadiennes (ESCC) ${ }^{16}$ pour l'Ontario et nous avons inclus les problèmes de santé suivants : hypertension, maladie cardiaque, asthme, AVC, diabète et cancer. Pour les groupes d'âge plus jeunes ( $<12$ ans), nous avons utilisé les estimations de Moran et coll ${ }^{17}$. Une des limites de l'ESCC pourrait être la sous-représentation d'individus appartenant à des populations socioéconomiquement défavorisées dans l'échantillon.

Les paramètres qui décrivent l'histoire naturelle et l'évolution clinique de l'infection proviennent d'études publiées (tableau 1, détails complets à l'annexe 1). La vitesse de propagation des épidémies dépend des taux de reproduction, soit le nombre d'infections secondaires causées par un premier cas infectieux. Avec une maladie pandémique contre laquelle personne n'est déjà immunisé, le taux de reproduction actuel correspond au taux de reproduction de base $\left(R_{0}\right)^{23}$. Pour saisir la variabilité de la transmission, particulièrement l'observation d'une surdispersion du taux de reproduction de base pour la COVID-19 due au fait que certains individus en sont des superpropagateurs, tandis que de nombreux autres la propagent beaucoup moins, nous avons ajouté la volatilité du taux de transmission ${ }^{24-26}$. Cela fait en sorte que chaque simulation donne un résultat différent en raison de la stochasticité (c.-à-d., variation aléatoire entre les simulations). Le modèle a été amorcé avec 750 cas prévalents (sur la base des 150 cas rapportés en Ontario le 19 mars 2020 et d'un taux présumé de signalement de $20 \%$ ) qui ont été distribués aléatoirement entre les compartiments infectieux. 


\section{Interventions}

Selon l'hypothèse, le dépistage faisait passer les individus présentant des symptômes bénins du compartiment infectieux au compartiment en isolement. Et les cas en isolement transmettaient moins l'infection que les cas en absence d'isolement. Selon l'hypothèse toujours, l'éloignement physique réduisait le nombre de contacts quotidiens dans la population entière. Les détails des paramètres variant selon les différentes interventions sont présentés au tableau 2. Pour le scénario de référence, nous avons présumé un niveau de dépistage et d'isolement en place et la mise en quarantaine d'une proportion des cas exposés. Nous avons ensuite ajouté d'autres mesures de contrôle : (i) dépistage accru et recherche des contacts; (ii) mesures strictes d'éloignement physique; et (iii) combinaison de dépistage accru et de recherche des contacts, associée à un éloignement physique moins strict qu'au numéro (ii). Nous avons tenu compte de 2 approches pour la mise en place des interventions : (i) durées fixes et (ii) approche dynamique cyclique en fonction du nombre de cas de soins intensifs dans la population. Lorsque les interventions n'étaient pas appliquées, les valeurs pour l'éloignement physique, le dépistage accru et la recherche des contacts revenaient au niveau du scénario de référence. Nous nous sommes concentrés sur la capacité des USI, étant donné qu'il pouvait s'agir de la ressource la plus limitée durant l'épidémie de COVID19. Avant l'émergence de la COVID-19, l'Ontario comptait environ 2000 lits d'USI (0,14 lit par 1000 habitants), mais $90 \%$ étaient occupés par des individus indemnes de la COVID-19. À la mi-mars 2020, le gouvernement de l'Ontario avait libéré 300 lits d'USI additionnels avec respirateurs (pour un total de 500 lits inoccupés (0,034 par 1000 habitants). À cet égard, nous avons modélisé 200 cas de COVID-19 aux USI (pour tout l'Ontario [0,014 par 1000]) comme seuil pour appliquer l'intervention. Cette valeur a été établie en fonction d'une saturation à environ $40 \%$ des lits disponibles, combinée à la reconnaissance du fait qu'il y a un écart

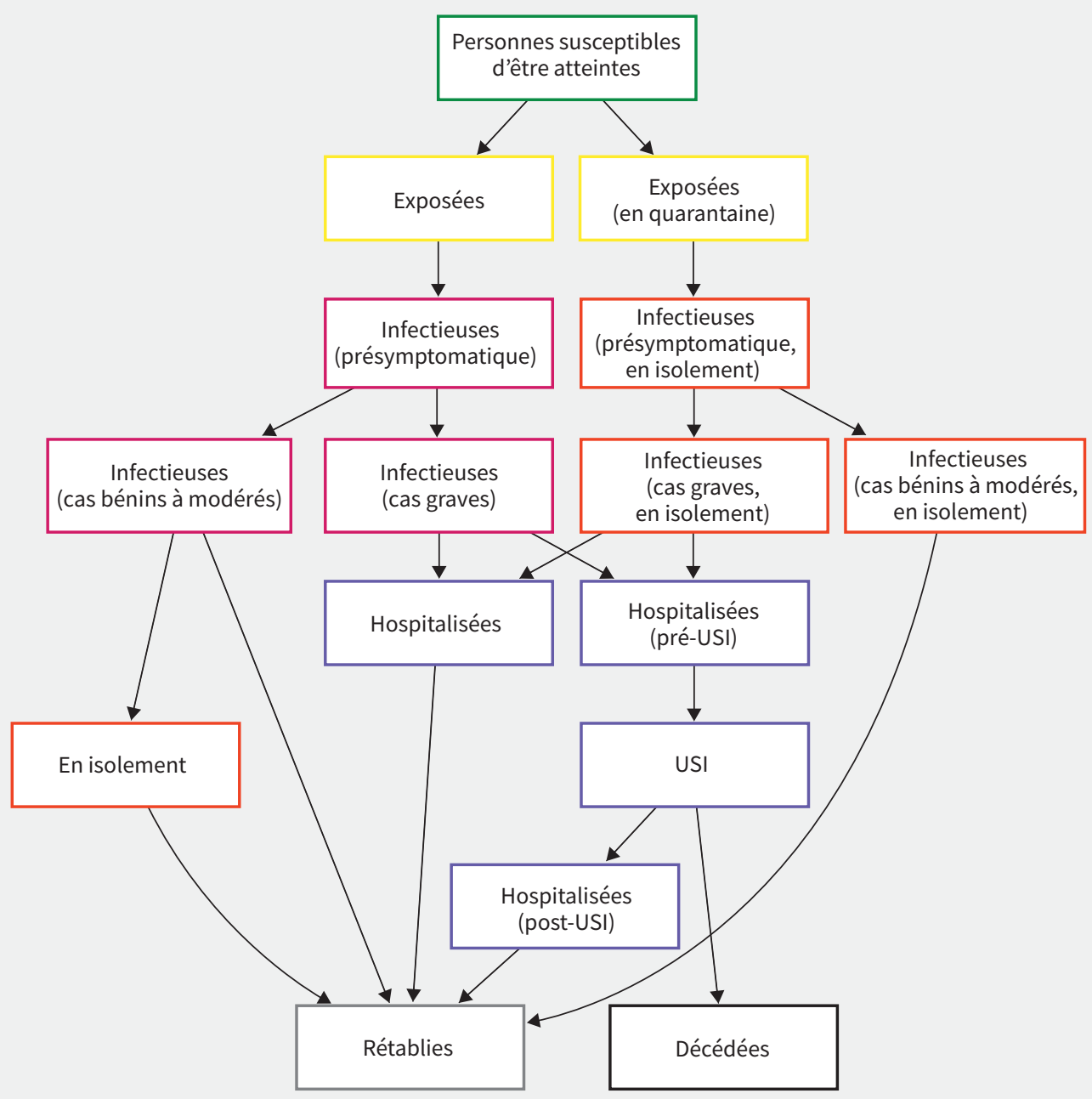

Figure 1 : Structure du modèle de transmission de la COVID-19. Les cas exposés peuvent être mis en quarantaine ou non; les cas en quarantaine représentaient ceux qui ont été identifiés lors de la recherche des contacts. Les cas hospitalisés sont présumés n'être plus infectieux pour autrui (en raison de la reconnaissance de l'infection) et sont inclus dans le modèle pour estimer les besoins en soins médicaux. Le modèle est stratifié par groupe d'âge et selon la présence ou l'absence de comorbidités. USI = unité de soins intensifs. 


\begin{tabular}{|c|c|c|c|c|c|}
\hline Paramètre & $\begin{array}{l}\text { Groupe } \\
\text { d'âge, ans }\end{array}$ & État de santé & Valeur & Détails & Source \\
\hline Période de latence, jours & Tous & Tous & 2.5 & Intervalle entre l'exposition et l'infectiosité & Références $18-20$ \\
\hline $\begin{array}{l}\text { Période infectieuse } \\
\text { présymptomatique, jours }\end{array}$ & Tous & Tous & 1 & $\begin{array}{l}\text { Durée de l'infectiosité avant l'apparition des } \\
\text { symptômes }\end{array}$ & Références $18-20$ \\
\hline $\begin{array}{l}\text { Période infectieuse } \\
\text { (cas bénins à modérés), jours }\end{array}$ & Tous & Tous & 6 & $\begin{array}{l}\text { Période infectieuse symptomatique des cas } \\
\text { bénins à modérés (en l'absence d'isolement) }\end{array}$ & Références $18-20$ \\
\hline $\begin{array}{l}\text { Période infectieuse } \\
\text { (cas grave), jours }\end{array}$ & Tous & Tous & 6 & $\begin{array}{l}\text { Période infectieuse symptomatique pour } \\
\text { l'infectiosité des cas graves; présumé égal à } \\
\text { l'intervalle avant hospitalisation }\end{array}$ & Références $18-20$ \\
\hline Taux de reproduction de base & Tous & Tous & 2.3 & $\begin{array}{l}\text { Nombre moyen d'infections secondaires } \\
\text { dérivées d'une infection primaire chez une } \\
\text { population susceptible d'être atteinte }\end{array}$ & Référence 6 \\
\hline Durée de la quarantaine, jours & Tous & Tous & 14 & Durée de la quarantaine des cas exposés & Politique actuelle \\
\hline $\begin{array}{l}\text { Risque relatif de transmission } \\
\text { par les cas en isolement }\end{array}$ & Tous & Tous & 0.1 & $\begin{array}{l}\text { Les cas en isolement sont présumés avoir } \\
\text { réduit la transmission par rapport aux cas non } \\
\text { reconnus }\end{array}$ & Hypothèse \\
\hline $\begin{array}{l}\text { Durée moyenne de } \\
\text { l'hospitalisation, soins } \\
\text { standards, jours }\end{array}$ & Tous & Tous & 10 & & Référence 21 \\
\hline $\begin{array}{l}\text { Durée moyenne de } \\
\text { l'hospitalisation, pré-USI, jours }\end{array}$ & Tous & Tous & 3 & $\begin{array}{l}\text { Pour les cas graves nécessitant des soins } \\
\text { intensifs }\end{array}$ & Référence 21 \\
\hline $\begin{array}{l}\text { Durée moyenne de } \\
\text { l'hospitalisation, USI, jours }\end{array}$ & Tous & Tous & 21 & $\begin{array}{l}\text { Pour les cas graves nécessitant des soins } \\
\text { intensifs }\end{array}$ & Référence 22 \\
\hline $\begin{array}{l}\text { Durée moyenne de } \\
\text { l'hospitalisation, post-USI, jours }\end{array}$ & Tous & Tous & 21 & $\begin{array}{l}\text { Pour les cas graves nécessitant des soins } \\
\text { intensifs }\end{array}$ & Référence 22 \\
\hline \multirow[t]{9}{*}{ Probabilité d'infection sévère } & & & & $\begin{array}{l}\text { Infections sévères nécessitant une } \\
\text { hospitalisation }\end{array}$ & Référence 21 \\
\hline & $<15$ & Sans comorbidités & 0,01 & & \\
\hline & $15-49$ & Sans comorbidités & 0,03 & & \\
\hline & $50-69$ & Sans comorbidités & 0.12 & & \\
\hline & $\geq 70$ & Sans comorbidités & 0,35 & & \\
\hline & $<15$ & Comorbidités & 0,02 & & \\
\hline & $15-49$ & Comorbidités & 0,06 & & \\
\hline & $50-69$ & Comorbidités & 0,25 & & \\
\hline & $\geq 70$ & Comorbidités & 0,76 & & \\
\hline $\begin{array}{l}\text { La gravité probable d'un cas } \\
\text { justifie l'admission à l'USI }\end{array}$ & Tous & Tous & 0,26 & & Référence 21 \\
\hline \multirow{9}{*}{$\begin{array}{l}\text { Probabilité de décès chez les } \\
\text { cas admis à l'USI }\end{array}$} & & & & & Référence 22 \\
\hline & $<15$ & Sans comorbidités & 0 & & \\
\hline & $15-49$ & Sans comorbidités & 0,2 & & \\
\hline & $50-69$ & Sans comorbidités & 0,36 & & \\
\hline & $\geq 70$ & Sans comorbidités & 0,58 & & \\
\hline & $<15$ & Comorbidités & 0 & & \\
\hline & $15-49$ & Comorbidités & 0,53 & & \\
\hline & $50-69$ & Comorbidités & 0,9 & & \\
\hline & $\geq 70$ & Comorbidités & 1 & & \\
\hline
\end{tabular}

Note : $\mathrm{SI}$ = soins intensifs, USI = unité de soins intensifs.

*Une description complète du modèle est fournie à l'annexe 1 (accessible ici en anglais : www.cmaj.ca/lookup/suppl/doi:10.1503/cmaj.200476/-/DC1). Le groupe d'âge et l'état de santé font référence aux groupes de population auxquelles la valeur du paramètre a été appliquée. 
Tableau 2 : Détails des scénarios du modèle*

\begin{tabular}{|c|c|c|c|c|c|}
\hline \multirow[b]{2}{*}{ Paramètre } & \multirow[b]{2}{*}{$\begin{array}{l}\text { Groupe d'âge, } \\
\text { ans }\end{array}$} & \multicolumn{4}{|c|}{ Scénario } \\
\hline & & $\begin{array}{l}\text { Scénario de } \\
\text { référence }\end{array}$ & $\begin{array}{l}\text { Dépistage } \\
\text { accru }\end{array}$ & $\begin{array}{l}\text { Éloignement } \\
\text { physique }\end{array}$ & $\begin{array}{l}\text { Dépistage accru } \\
\text { avec éloignement } \\
\text { physique limité } \\
\text { (combinaison) }\end{array}$ \\
\hline \multirow[t]{3}{*}{ Cas non en quarantaine, testés et en isolement, \% } & $<15$ & 10 & 40 & 10 & 40 \\
\hline & $15-49$ & 40 & 60 & 40 & 60 \\
\hline & $\geq 50$ & 70 & 80 & 70 & 80 \\
\hline Cas exposés, en quarantaine avant infectiosité, \% & Tous & 10 & 30 & 10 & 30 \\
\hline $\begin{array}{l}\text { Réduction des contacts par l'éloignement } \\
\text { physique, \% }\end{array}$ & Tous & 0 & 0 & 60 & 25 \\
\hline
\end{tabular}

entre les cas qui contractent l'infection et ceux qui nécessitent des soins intensifs, faisant en sorte que l'on pouvait s'attendre à ce que les besoins en USI augmentent rapidement une fois que les premiers cas de COVID-19 consultaient pour des soins.

\section{Paramètres}

Les paramètres clés du modèle incluaient les taux d'attaque épidémique (\% de la population atteinte à la fin de la période de 2 ans), la prévalence des hospitalisations, le recours aux USI et les décès. À titre de comparaison, nous montrons la capacité maximale et actuelle des USI par 1000 habitants par rapport aux projections du modèle. Pour les scénarios d'interventions dynamiques, nous avons aussi calculé leur durée d'application pendant la période du modèle, soit 2 ans, comme mesure de leur intensité. Nous présentons les paramètres du modèle sous forme de médianes et d'intervalles de crédibilité de 100 expériences répliqués par intervention; les intervalles de crédibilité de $95 \%$ représentent l'éventail des paramètres allant du $2,5^{\mathrm{e}}$ percentile au $97,5^{\mathrm{e}}$ percentile, pour toutes les expériences répliquées.

\section{Approbation éthique}

Étant donné que cette étude reposait sur l'utilisation de données agrégées accessibles au public, l'approbation d'un comité d'éthique de la recherche n'était pas nécessaire.

\section{Résultats}

\section{Scénario de référence}

Dans le scénario de référence du modèle, avec le dépistage limité, l'isolement et la mise en quarantaine, nous avons estimé que $56 \%$ (intervalle de crédibilité de $95 \%$, $42 \%-63 \%$ ) de la population ontarienne contractait l'infection au cours de l'épidémie, y compris les cas les plus bénins aux plus graves. Selon l'hypothèse, les taux d'attaque étaient les plus élevés chez les individus de 5 à 14 ans (77\%, intervalle de crédibilité de $95 \%$, $63 \%-83 \%$ ) et de 15-49 ans (63\%, intervalle de crédibilité de
$95 \%, 48 \%-71 \%)$. Des taux d'attaque moindres étaient projetés chez les enfants de moins de 5 ans (50\%, intervalle de crédibilité de $95 \%, 37 \%-58 \%$ ), les adultes de 50 à 69 ans (47\%, intervalle de crédibilité de $95 \%, 34 \%-55 \%$ ) et de 70 ans et plus (30\%, intervalle de crédibilité de $95 \%, 21 \%-36 \%)$. Un exemple de la trajectoire de l'éclosion dans les simulations du modèle est présenté à la figure 2. Au moment du sommet épidémique, en l'absence de toute contrainte au plan des ressources médicales (en supposant que tous les cas qui nécessitent des soins les reçoivent), nous avons projeté 107000 (intervalle de crédibilité de $95 \%, 60760-149000$ ) cas hospitalisés (soins standards) et 55500 (intervalle de crédibilité de $95 \%$, 32700-75200) cas dans les USI. La forte prévalence du recours aux USI fait écho à la durée moyenne des séjours dans les USI pour la COVID-19 dans d'autres pays.

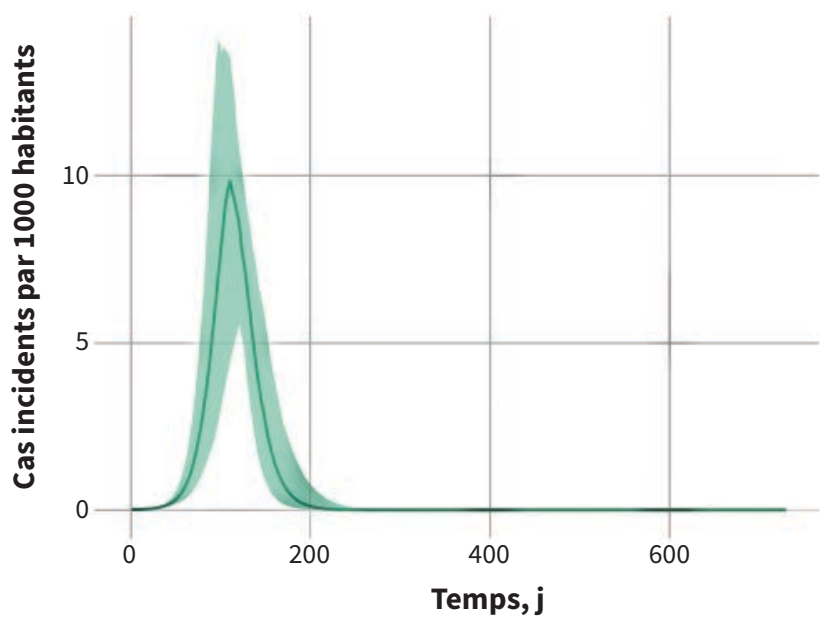

Figure 2 : Trajectoire projetée de l'épidémie de COVID-19 pour le modèle du scénario de référence avec intervention minimale. Les cas quotidiens incidents par 1000 habitants sont présentés. La ligne représente la valeur médiane de 100 simulations du modèle et la zone ombrée indique l'intervalle de crédibilité de $95 \%$. 


\section{Interventions à durée fixe}

Selon les projections, toutes les interventions envisagées retardaient le sommet épidémique et réduisaient le nombre de cas nécessitant des soins intensifs au moment du sommet épidémique (figure 3). L'efficacité des interventions est proportionnelle à leur durée. Pour toutes les interventions, lorsque leur durée était de 6 mois ou moins, on ne percevait pas de différence appréciable quant au taux d'attaque final. Avec une durée de 12 et de 18 mois de mesures plus strictes, la proportion de la population atteinte était réduite à la fin de la période de 2 ans, et dans certaines simulations, la prévalence des cas nécessitant des soins intensifs diminuait en deçà de la capacité actuelle de l'Ontario pour toute la période ou une partie. L'effet le plus marqué a été observé avec l'éloignement physique plus strict. Selon les projections, les interventions combinées de dépistage accru et d'éloignement physique moins strict réduisaient substantiellement les taux d'attaque lorsqu'elles étaient appliquées pendant 18 mois, tandis qu'en moyenne, le dépistage accru en l'absence de mesures d'éloignement physique avait un effet plus modeste. On a observé une variabilité substantielle des projections du modèle due à sa stochasticité.

\section{Interventions dynamiques cycliques}

Nous avons aussi exploré les interventions dynamiques cycliques adaptées aux fluctuations de l'épidémie. Selon les projections, avec des durées d'éloignement physique potentiellement plus brèves qu'avec l'approche à durée fixe, les interventions dynamiques réduisaient efficacement la proportion de la population atteinte à la fin de la période de 2 ans (figure 4). Par exemple, appliqués de manière dynamique et cyclique, 13 mois d'éloignement physique réduisaient de $2 \%$ le taux d'attaque global

A

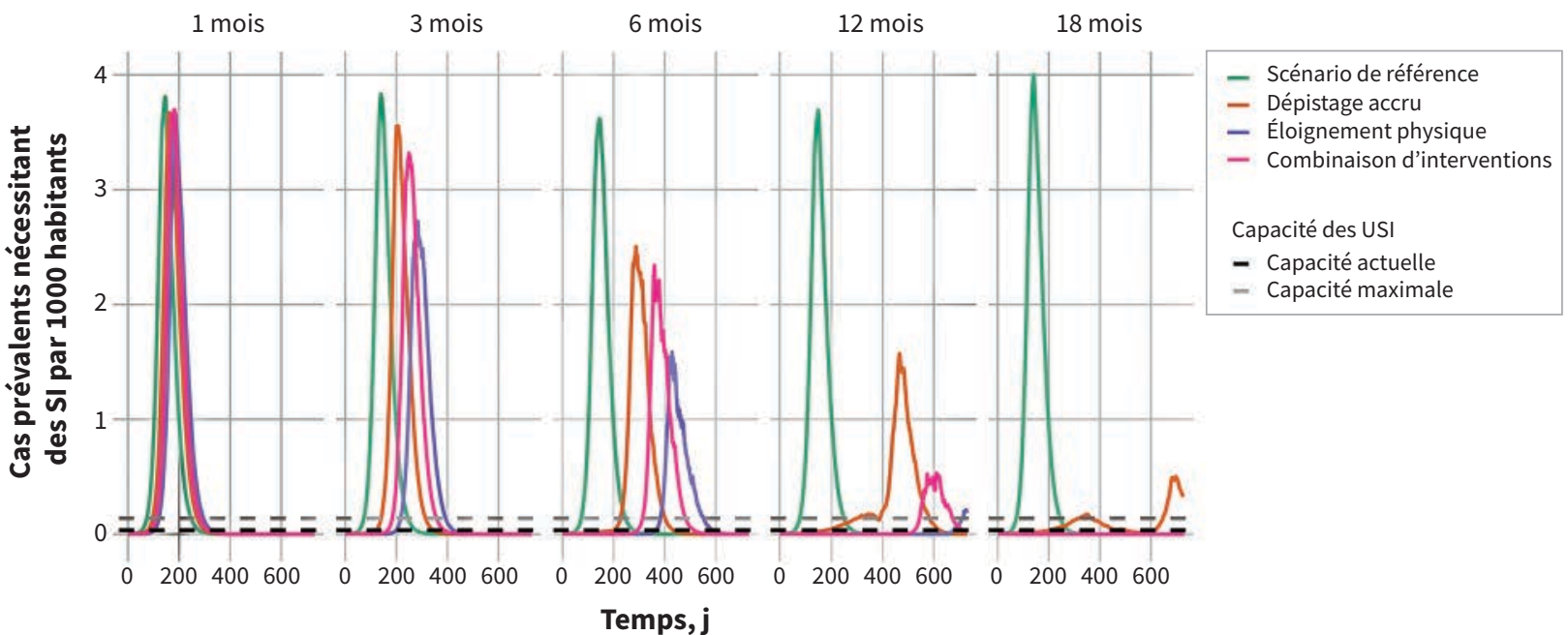

B

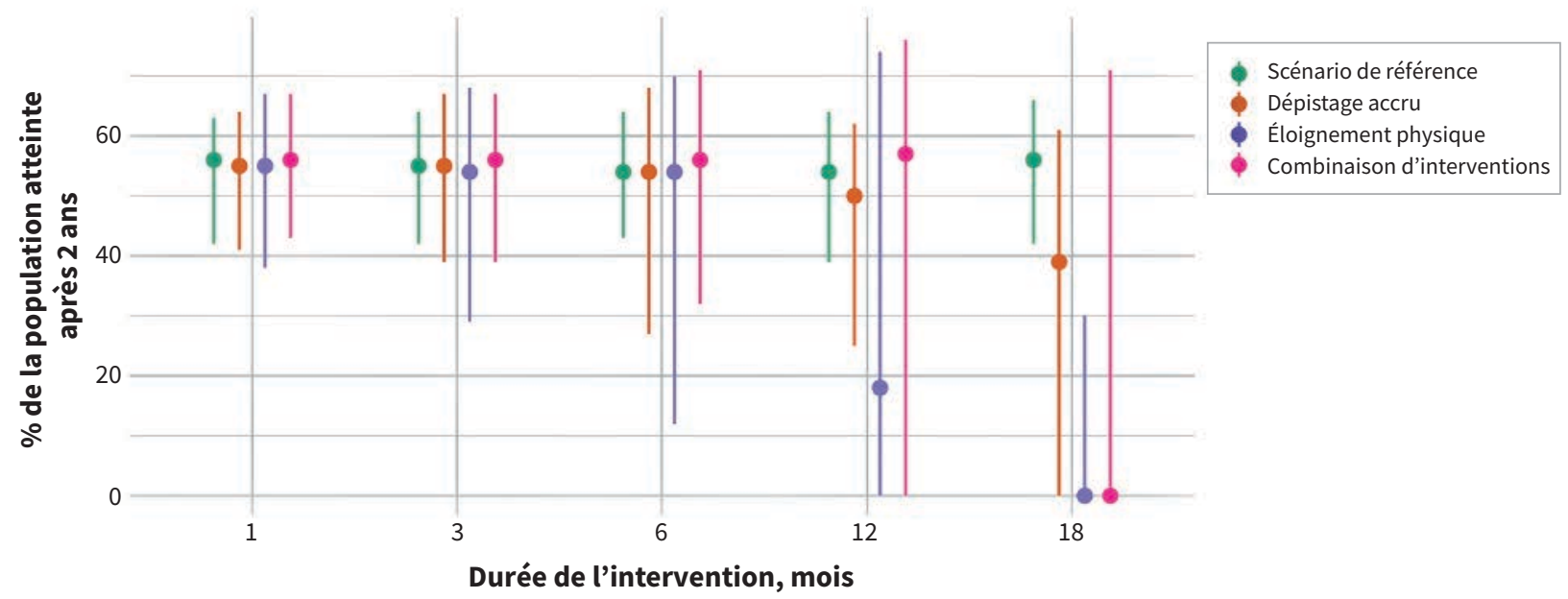

Figure 3 : Besoins projetés en lits de soins intensifs (SI) et taux d'attaque pour les interventions à durée fixe. (A) Les cas prévalents nécessitant des SI sont montrés pour des durées d'interventions de 1, 3, 6, 12 et 18 mois. La capacité maximale et actuelle des unités de soins intensifs (USI) en Ontario est indiquée par les lignes horizontales discontinues. Les médianes sont présentées. (B) Pourcentage projeté de la population atteinte sur une période de 2 ans selon le modèle. Les taux d'attaque incluent toutes les infections, indépendamment de leur gravité. Noter que la légère variabilité de la taille de l'épidémie pour le scénario de référence (sans intervention additionnelle) est le reflet de la stochasticité du modèle entre les simulations. Des durées d'éloignement physique extrêmes créent la possibilité d'une extinction stochastique (" épuisement ») de l'épidémie. 
médian. Avec l'éloignement physique seul et les scénarios d'interventions combinées, nous avons observé des courbes épidémiques atypiques, le nombre de cas augmentant et diminuant en alternance au fil du temps. Dans ces scénarios, le nombre médian de cas dans les USI a diminué en deçà des estimations actuelles de la capacité des USI de l'Ontario.

\section{Interprétation}

La COVID-19 pose un défi inédit aux sociétés. Alors que la maladie grave, particulièrement chez les individus âgés, est suffisamment fréquente pour excéder la capacité des USI dans une société27, la maladie bénigne non reconnue (particulièrement
A

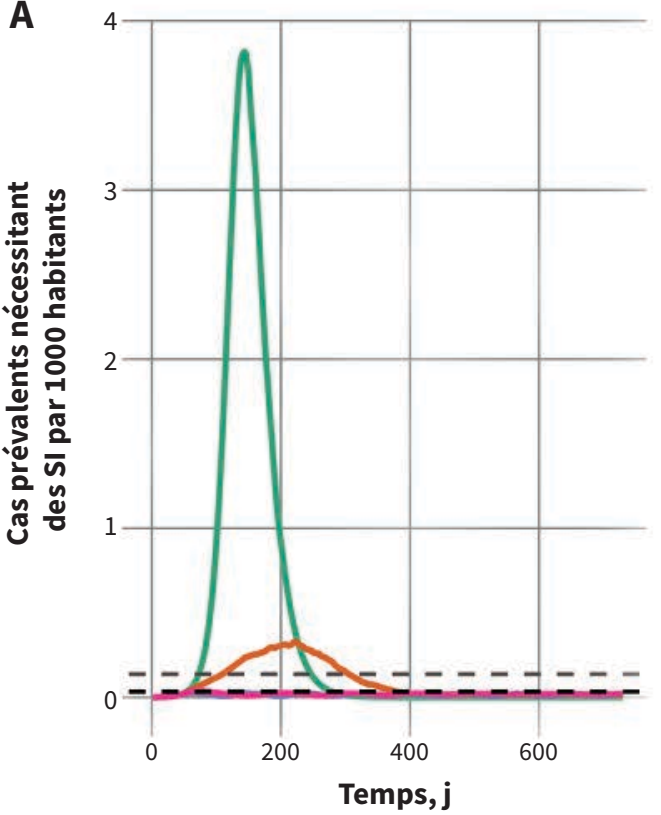

B

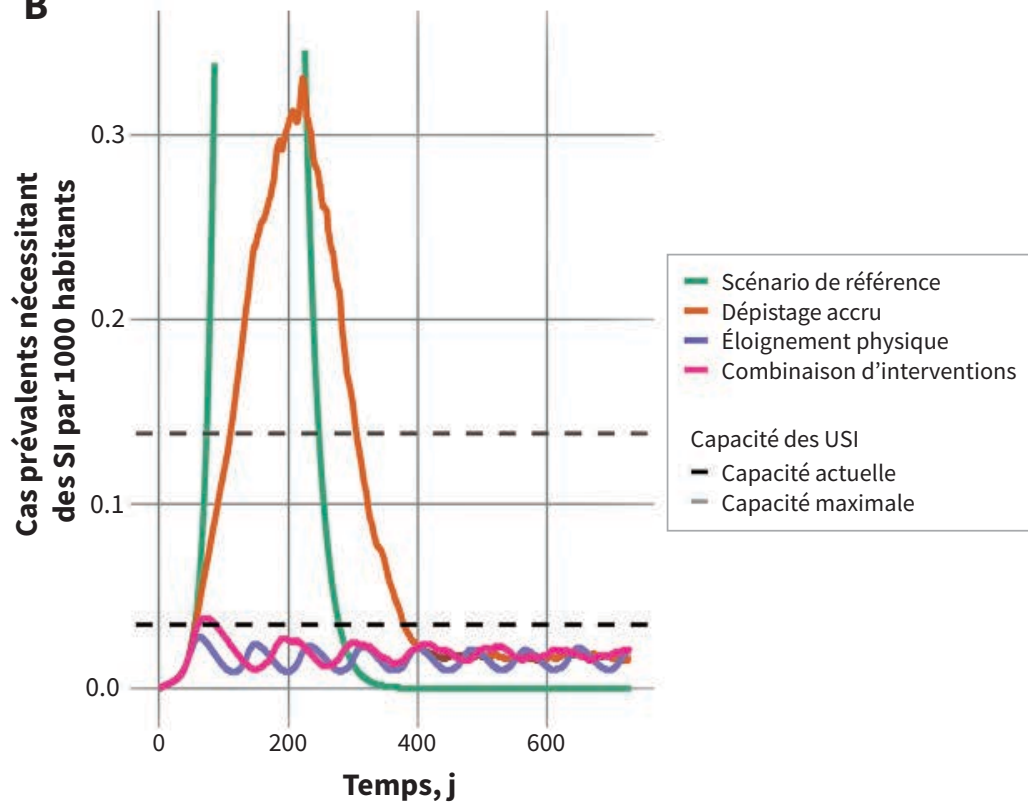

C

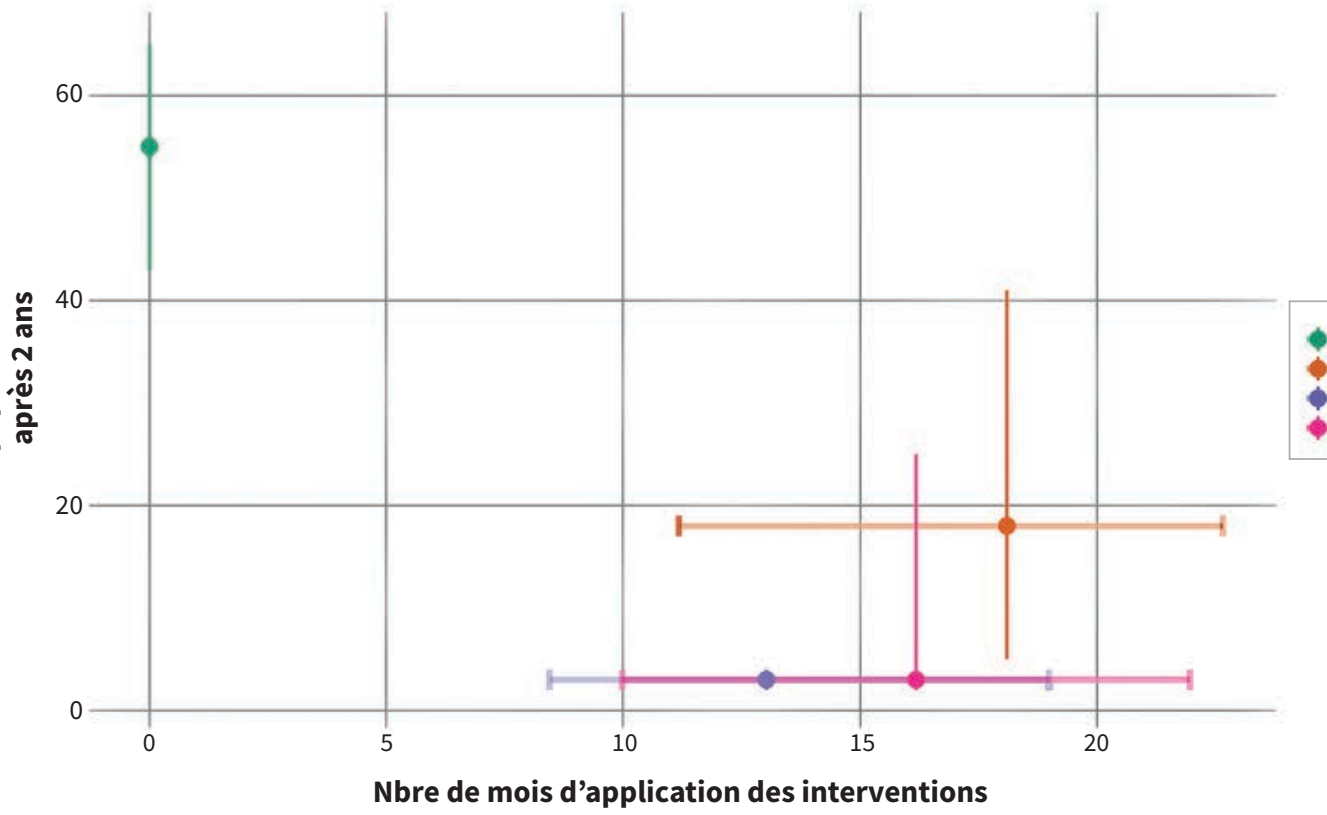

Scénario de référence

Dépistage accru

Éloignement physique

Combinaison d'interventions

Figure 4 : Besoins projetés en lits de soins intensifs (SI) et taux d'attaque pour les interventions dynamiques. (A) Les cas prévalents nécessitant des SI sont montrés pour le scénario de référence et pour 3 scénarios d'intervention. Les interventions sont appliquées cycliquement (et reviennent aux valeurs des paramètres du scénario de référence), selon le nombre de cas de COVID-19 dans les unités de soins intensifs (USI). La capacité maximale et actuelle des USI en Ontario est indiquée par les lignes horizontales discontinues. Les médianes sont présentées. (B) Vue agrandie des cas prévalents dans les USI pour montrer la dynamique pour l'éloignement physique strict et les scénarios combinés. (C) Estimations projetées du pourcentage de la population atteinte sur une période de 2 ans selon le modèle. Les taux d'attaque incluent toutes les infections incidentes, indépendamment de leur gravité. La durée de l'application des interventions dynamiques est montrée en abscisse. Les points indiquent la durée médiane et les lignes, les intervalles de crédibilité de $95 \%$ pour chaque scénario. 
chez les individus jeunes) contribue à sa transmission ${ }^{28}$, et des éclosions risquent de n'être identifiées que lors de superpropagations ${ }^{25}$, souvent dans des milieux comme les établissements de santé ${ }^{26}$. Par opposition au syndrome respiratoire aigu sévère $(\mathrm{SRAS})^{29}$, la fréquence élevée de cas bénins signifie que les stratégies uniquement axées sur le dépistage et l'isolement risquent d'être impuissantes à prévenir la propagation de l'épidémie et de surcharger notre système de santé26. C'est pourquoi malgré leurs coûts connexes, des interventions à l'échelle des populations ont été utilisées afin de prévenir l'effondrement des systèmes de santé $^{30}$. Même si les événements survenus en Chine, à Singapour, à Hong Kong et ailleurs ont montré qu'il est possible de contenir l'épidémie de COVID-1930-33, son essaimage dans d'autres pays du monde aux systèmes de santé défaillants ${ }^{34}$, signifie que la COVID-19 continuera de ressurgir pendant un certain temps. Tandis que les mesures de confinement efficaces maintiennent un nombre important de personnes susceptibles d'être atteintes dans les populations, le risque de résurgence de l'épidémie persistera tant qu'un vaccin contre la COVID-19 n'aura pas été conçu et fabriqué à grande échelle, ou tant que d'importantes fractions de la population n'auront pas été atteintes, avec ce que cela suppose de décès ou d'immunisations ${ }^{5}$.

Les stratégies de contrôle de la COVID-19 doivent donc jongler avec des risques opposés : d'une part, risques de mortalité et d'effondrement du système de santé, et de l'autre, risques économiques, perturbations et conséquences pour la santé. Nous avons évalué ici des stratégies plausibles pour atténuer l'épidémie de COVID-19 en Ontario, au Canada. Nous nous sommes concentrés sur les ressources des USI pour 2 raisons : premièrement, parce que cet élément de la plupart des systèmes de santé représente une ressource limitée qui est sujette à saturation; et deuxièmement, parce qu'une telle saturation donne lieu à des augmentations soudaines des taux de létalité, car les individus atteints du syndrome de détresse respiratoire aigu décéderont rapidement faute d'accès à la ventilation mécanique. De manière générale, nous trouvons que l'éloignement physique prolongé est la stratégie à privilégier pour maintenir les ressources des USI, mais une durée fixe extrême d'éloignement physique est nécessaire pour empêcher l'épidémie de paralyser les USI. Cela dit, même sans réduire la taille de l'éclosion dans son ensemble, l'éloignement physique comporte l'avantage additionnel de retarder le sommet de l'épidémie, ce qui donne du temps pour développer la capacité des systèmes de santé et trouver des traitements et des vaccins. Les sociétés restent toutefois vulnérables aux résurgences dans la mesure où une fraction critique de la population reste vulnérable à la maladie (on évalue cette fraction à environ $1 / R_{0}$ ). Il faudra procéder à des études séroépidémiologiques pour suivre l'évolution de la vulnérabilité avec le temps. Et de telles études n'ont pas encore été réalisées au Canada.

Par opposition à l'éloignement physique à durée fixe, nous trouvons qu'à partir d'un seuil donné, l'éloignement physique dynamique cyclique adaptée aux besoins et basée sur la capacité des USI représente une stratégie de contrôle plus efficace et peut-être plus acceptable. L'éloignement physique peut être relâché, puis réinstauré quand, inévitablement, son relâchement entraîne une résurgence de la maladie dans la population. Elle permet effectivement de maintenir la capacité des USI et réduit considérablement les taux d'attaque dans leur ensemble, tout en abrégeant la durée totale de l'éloignement physique, comparativement à une stratégie à durée fixe d'efficacité comparable. Cela peut sembler contre-intuitif, mais selon une piste intéressante fournie par notre modèle, les interventions dynamiques peuvent être réactivées et se révéler très efficaces lorsque les éclosions qui ressurgissent sont encore de faible ampleur.

En outre, l'éloignement physique dynamique pourrait permettre aux populations et à l'économie de « respirer » par intervalles, ce qui rend cette stratégie plus viable. Nous avons aussi constaté que dans le scénario dynamique, une approche d'éloignement physique moins strict combinée à un dépistage accru, à l'isolement et à la mise en quarantaine pourrait avoir un effet similaire à celui de l'éloignement physique plus strict utilisé seul. Il est plausible qu'avec l'augmentation de la capacité de dépistage, une approche combinée reposant moins sur l'éloignement physique permettra d'arriver à un juste équilibre entre maîtrise de la maladie et degré de perturbation sociale ${ }^{36}$.

Un étalonnage en fonction des données réelles d'éloignement physique est possible ${ }^{37}$ et c'est un domaine pour la recherche future. Toutefois, de manière générale, des mesures d'éloignement physique moins strictes pourraient reposer sur le libre arbitre et l'autorisation de petits rassemblements, tandis que les interventions plus strictes impliqueraient le « confinement » des personnes en isolement à la maison et s'exposant à des sanctions d'ordre juridique si elles sortent sans raison valable ${ }^{38}$. Éventuellement, ce type d'interventions visant à contrôler la maladie doit être combiné à des systèmes de surveillance accrue, qui permettent d'apporter les ajustements requis en fonction des données observées.

\section{Limites de l'étude}

Au moment d'écrire ces lignes, étant donné les limites bien documentées de la capacité de dépistage en Ontario ${ }^{39}$ et le manque de données sur l'occupation des lits d'USI par les patients atteints de la COVID-19, il était difficile de savoir où on se situe sur la courbe épidémique actuellement. Les défis relatifs à l'étendue et à la vitesse du dépistage ${ }^{39}$ font du nombre de cas un paramètre plus ou moins fiable de l'activité sous-jacente de la maladie. Il a été difficile d'obtenir les indispensables données de la province sur l'occupation des USI, quoiqu'elles aient récemment été publiées dans des rapports d'enquête ${ }^{40}$. Fait à noter, cette version de notre modèle n'inclut pas les cycles de transmission dans les hôpitaux. Les cas de transmission dans les établissements de santé ont le potentiel de réduire rapidement et considérablement la capacité des USI, car des infirmières, des médecins et des inhalothérapeutes de métier pourraient contracter le virus et devoir se retirer; les USI seraient alors vite surchargées de nouveaux patients (travailleurs de la santé contaminés et patients admis pour d'autres raisons ayant contracté la COVID-19 à l'hôpital).

Dans tout modèle, il faut équilibrer pragmatisme et réalité. Ici, nous n'avons pas tenté de modéliser les mesures d'éloignement physique de façon hautement réaliste, mais plutôt de 
manière générique, dans le but de réduire la fréquence des contacts. Notre compréhension de l'histoire naturelle de l'infection au SRAS-CoV-2 continue d'évoluer et son mode précis de transmission aux stades présymptomatique et sous-clinique demeure incertain. Face à l'incapacité de dénombrer les cas avec certitude, notamment en raison des cas asymptomatiques ou bénins, l'éloignement physique revêt plus d'importance comme mesure de contrôle.

Nous ne formulons aucune suggestion politique précise sur la façon d'atteindre les réductions associées à l'éloignement physique modélisées ici, et nous ne considérons pas ce modèle comme une simulation réaliste de la situation actuelle en Ontario. À cet égard, il est préférable d'interpréter notre modèle sur une base qualitative, plutôt que quantitative.

Le modèle ne tient pas compte des saisons; il est possible que la transmission diminue pendant l'été ${ }^{41}$ et s'accompagne d'une baisse de cas puis, qu'elle ressurgisse au retour de la saison froide. Même si les limites inhérentes à notre modèle sont une source d'incertitude, de dernier fournit aux décideurs et aux gestionnaires des pistes qualitatives quant au rôle de l'éloignement physique, quant aux durées relativement longues des interventions requises pour contrôler la courbe épidémique et quant à l'utilisation potentielle d'interventions cycliques afin de les aider à établir les meilleures approches pour contrôler l'épidémie au cours des mois à venir, en tenant compte des données empiriques générées par d'autres pays, ailleurs dans le monde.

En terminant, nous n'avons pas modélisé le fait que les augmentations abruptes de décès découlant de l'incapacité des USI à admettre d'autres patients entraîneraient une baisse de la demande de ces lits. Notre objectif ici est d'éclairer les politiques pour éviter une telle issue dans la mesure du possible.

\section{Conclusion}

Nous avons modélisé les contours plausibles de l'épidémie de COVID-19 en Ontario, au Canada en nous concentrant sur la préservation des ressources des USI. En l'absence d'éloignement physique substantiel ou d'une combinaison d'éloignement physique modérée, de dépistage accru et d'isolement, nous projetons que les ressources des USI seraient rapidement submergées; cette conclusion concorde avec d'autres modélisations ${ }^{12}$ et avec la situation actuelle en Italie et en Espagne. Sur une note plus positive, nous projetons que l'éloignement physique dynamique, qui s'adapte aux fluctuations de l'occupation des USI, pourrait préserver la capacité du système de santé, en plus de donner un répit psychologique et économique périodique aux populations.

\section{Références}

1. Novel coronavirus (2019-nCoV) situation report - 1: 21 January 2020. Genève : Organisation mondiale de la santé; 2020. Accessible ici : www.who.int/docs/ default-source/coronaviruse/situation-reports/20200121-sitrep-1-2019-ncov.pdf (consulté le 22 mars 2020).

2. Coronavirus disease (COVID-19) situation report - 63. Genève : Organisation mondiale de la santé; le 23 mars 2020. Accessible ici : www.who.int/docs/ default-source/coronaviruse/situation-reports/20200323-sitrep-63-covid-19.pdf ?sfvrsn=d97cb6dd_2 (consulté le 24 mars 2020).

3. Epidemiological data from the COVID-19 outbreak in Canada. COVID-19 Canada Open Data Working Group; 2020. Accessible ici : https://github.com/ishaberry/ Covid19Canada (consulté le 22 mars 2020).
4. Report of the WHO-China Joint Mission on Coronavirus disease 2019 (COVID-19). Genève : Organisation mondiale de la santé; le 28 février 2020. Accessible ici : www.who.int/publications-detail/report-of-the-who-china-joint-mission-on -coronavirus-disease-2019-(covid-19) (consulté le 22 mars 2020).

5. The Novel Coronavirus Pneumonia Emergency Response Epidemiology Team. The epidemiological characteristics of an outbreak of 2019 novel coronavirus disease (COVID-19) - China, 2020. China CDC Weekly 2020;2:113-22.

6. Li Q, Guan X, Wu P, et al. Early transmission dynamics in Wuhan, China, of novel coronavirus-infected pneumonia. N Engl J Med 2020;382:1199-1207.

7. Kucharski AJ, Russell TW, Diamond C, et al. Early dynamics of transmission and control of COVID-19: a mathematical modelling study. Lancet Infect Dis 2020 le 11 mars; Mar 11. pii: S1473-3099(20)30144-4. doi: 10.1016/S1473-3099 (20)30144-4. [Cyberpublication avant impression].

8. Wu JT, Leung K, Bushman M, et al. Estimating clinical severity of COVID-19 from the transmission dynamics in Wuhan, China. Nat Med 2020 le 19 mars. doi: 10.1038/s41591-020-0822-7.

9. Weiss P, Murdoch DR. Clinical course and mortality risk of severe COVID-19. Lancet 2020;395:1014-5.

10. Ferguson NM, Cummings DA, Fraser C, et al. Strategies for mitigating an influenza pandemic. Nature 2006;442:448-52.

11. Henry B. Canadian Pandemic Influenza Preparedness (CPIP) Task Group. Canadian pandemic influenza preparedness: health sector planning guidance. Can Commun Dis Rep 2018;44:6-9.

12. Ferguson NM, Laydon D, Nedjati-Gilani G, et al. Impact of non-pharmaceutical interventions (NPIs) to reduce COVID-19 mortality and healthcare demand. London (UK) Imperial College COVID-19 Response Team; 2020 le 16 mars. Accessible ici : www.imperial.ac.uk/media/imperial-college/medicine/sph/ide/ gida-fellowships/Imperial-College-COVID19-NPI-modelling-16-03-2020.pdf (consulté le 22 mars 2020).

13. R Core Team. R: A language and environment for statistical computing. R Foundation for Statistical Computing, Vienne, Autriche.

14. Table 17-10-0005-01. Population estimates on July 1st, by age and sex. Ottawa : Statistique Canada; 2019.

15. Mossong J, Hens N, Jit M, et al. Social contacts and mixing patterns relevant to the spread of infectious diseases. PLoS Med 2008;5:e74.

16. Canadian Community Health Survey, public use microdata files. Cat no 82M0013X. Ottawa : Statistique Canada; 2016. Accessible ici : www150.statcan. gc.ca/n1/en/catalogue/82M0013X (consulté le 15 mars 2020).

17. Moran K, Maaten S, Guttmann A, et al. Influenza vaccination rates in Ontario children: implications for universal childhood vaccination policy. Vaccine 2009;27:2350-5.

18. Bi Q, Wu Y, Mei S, et al. Epidemiology and transmission of COVID-19 in Shenzhen China: analysis of 391 cases and 1286 of their close contacts. medRxiv 2020 le 27 mars. doi: 10.1101/2020.03.03.20028423.

19. Tindale L, Coombe M, Stockdale J, et al. Transmission interval estimates suggest pre-symptomatic spread of COVID-19. medRxiv 2020 le 6 mars. doi: 10.1101/2020.03.03.20029983.

20. Woelfel R, Corman V, Guggemos W, et al. Clinical presentation and virological assessment of hospitalized cases of coronavirus disease 2019 in a travel-associated transmission cluster. medRxiv 2020 le 8 mars. [Cyberpublication avant impression]. doi: $10.1101 / 2020.03 .05 .20030502$.

21. Wang D, Hu B, Hu C, et al. Clinical characteristics of 138 hospitalized patients with 2019 novel coronavirus-infected pneumonia in Wuhan, China. JAMA 2020 le 7 février. [Cyberpublication avant impression]. doi: 10.1001/jama.2020.1585.

22. Yang $X, Y u$ Y, Xu J, et al. Clinical course and outcomes of critically ill patients with SARS-CoV-2 pneumonia in Wuhan, China: a single-centered, retrospective, observational study. Lancet Respir Med 2020 le 24 février. [Cyberpublication avant impression]. pii: S2213-2600(20)30079-5. doi: 10.1016/S2213-2600(20)30079-5.

23. Pandemic Influenza Outbreak Research Modelling Team (Pan-infORM); Fisman D. Modelling an influenza pandemic: a guide for the perplexed. CMAJ 2009;181:171-3.

24. Camacho A, Kucharski A, Aki-Sawyerr Y, et al. Temporal changes in Ebola transmission in Sierra Leone and implications for control requirements: a realtime modelling study. PLoS Curr 2015 le 10 février;7. pii: ecurrents.outbreaks.4 06ae55e83ec0b5193e30856b9235ed2.

25. Liu Y, Eggo RM, Kucharski AJ. Secondary attack rate and superspreading events for SARS-CoV-2. Lancet 2020;395:e47.

26. Frieden TR, Lee CT. Identifying and interrupting superspreading events implications for control of severe acute respiratory syndrome coronavirus 2 . Emerg Infect Dis 2020 le 18 mars. [Cyberpublication avant impression] doi: 10.3201/eid2606.200495. 
27. Cereda D, Tirani M, Rovida F, et al. The early phase of the COVID-19 outbreak in Lombardy, Italy. Accessible ici : https://arxiv.org/ftp/arxiv/ papers/2003/2003 .09320.pdf (consulté le 24 mars 2020).

28. Li R, Pei S, Chen B, et al. Substantial undocumented infection facilitates the rapid dissemination of novel coronavirus (SARS-CoV2). Science 2020 le 16 mars. [Cyberpublication avant impression]. pii: eabb3221. doi: 10.1126/science. abb3221.

29. Lipsitch M, Cohen T, Cooper B, et al. Transmission dynamics and control of severe acute respiratory syndrome. Science 2003;300:1966-70.

30. Wang C, Liu L, Hao X, et al. Evolving epidemiology and impact of non-pharmaceutical interventions on the outbreak of Coronavirus disease 2019 in Wuhan, China. medRxiv 2020 le 6 mars. doi: 10.1101/2020.03.03.20030593.

31. Koo J, Cook AR, Park M, et al. Interventions to mitigate early spread of SARSCoV-2 in Singapore: a modelling study. Lancet Infect Dis 2020 le 23 mars. [Cyberpublication avant impression]. doi: 10.1016/S1473-3099(20)30162-6.

32. Cowling BJ, Ali ST, Ng TWY, et al. Impact assessment of non-pharmaceutical interventions against COVID-19 and influenza in Hong Kong: an observational study. medRxiv 2020 le 16 mars. doi: 10.1101/2020.03.12.20034660.

33. Lai S, Ruktanonchai NW, Zhou L, et al. Effect of non-pharmaceutical interventions for containing the COVID-19 outbreak in China. medRxiv 2020 le 13 mars. [Cyberpublication avant impression]. doi: 10.1101/2020.03.03.20029843.

34. Tuite AR, Bogoch, II, Sherbo R, et al. Estimation of Coronavirus disease 2019 (COVID-19) burden and potential for international dissemination of infection from Iran. Ann Intern Med 2020 le 16 mars. doi: 10.7326/M20-0696.
35. Pandemic Influenza Outbreak Research Modelling Team. Fisman D. Modelling an influenza pandemic: A guide for the perplexed. CMAJ 2009;181:171-3.

36. Lee VJ, Chiew CJ, Khong WX. Interrupting transmission of COVID-19: lessons from containment efforts in Singapore. J Travel Med 2020 le 13 mars. [Cyberpublication avant impression]. pii: taaa039. doi: 10.1093/jtm/taaa039.

37. Thompson S, Serkez Y, Kelley L. How has your state reacted to social distancing? The New York Times 2020 le 23 mars. Accessible ici : www.nytimes.com/interactive/ 2020/03/23/opinion/coronavirus-economy-recession.html (consulté le $1^{\text {er }}$ avril 2020).

38. Coronavirus disease 2019 (COVID-19) pandemic: increased transmission in the EU/EEA and the UK — seventh update: 2020 le 25 mars. Sweden: European Centre for Disease Prevention and Control; 2020. Accessible ici : www.ecdc.europa.eu/ sites/default/files/documents/RRA-seventh-update-Outbreak-of-coronavirus -disease-COVID-19.pdf (consulté le $1^{\text {er }}$ avril 2020).

39. Russell A, Cain P. Coronavirus: Ontario lags behind all other provinces in COVID-19 testing. Global News; mise à jour : 2020 le 31 mars. Accessible ici : https://globalnews.ca/news/6752239/coronavirus-ontario-behind-covid-19-testing (consulté le $1^{\mathrm{er}}$ avril 2020).

40. Crawley M. Ontario intensive care units see jump in COVID-19 patients. CTV News; mise à jour: 2020 le 28 mars. Accessible ici : www.cbc.ca/news/canada/ toronto/covid-19-ontario-hospital-intensive-care-icu-patients-1.5512926 (consulté le $1^{\mathrm{er}}$ avril 2020).

41. Kissler SM, Tedijanto C, Goldstein E, et al. Projecting the transmission dynamics of SARS-CoV-2 through the post-pandemic period. medRxiv 2020 le 6 mars. doi: 10.1101/2020.03.04.20031112.
Intérêts concurrents : Amy Greer reçoit un financement du Conseil de recherches en sciences naturelles et en génie du Canada, des Instituts de recherche en santé du Canada et du Programme des chaires de recherche du Canada. Aucun autre intérêt concurrent n'a été déclaré.

Cet article a été révisé par des pairs.

Affiliations : École Dalla Lana de santé publique (Tuite, Fisman), Université de Toronto Toronto, Ont.; Département de médecine des populations (Greer), Université de Guelph, Guelph, Ont..

Collaborateurs : Tous les auteurs ont contribué à la conception et à l'élaboration du travail, à l'acquisition, à l'analyse et à l'interprétation des données, ainsi qu'à la rédaction de l'ébauche et à sa révision critique. Tous les auteurs ont donné leur approbation finale pour la version soumise pour publication et assument l'entière responsabilité de tous les aspects du travail.

Financement : La recherche a bénéficié de l'appui d'une subvention des IRSC (Financement canadien pour une intervention de recherche rapide contre la COVID-19 OV4-170360) versée à David Fisman.

Partage des données : Toutes les données utilisées pour l'établissement des paramètres de ce modèle se trouvent dans le domaine public et sont accessibles par le biais des références citées dans le manuscrit et l'annexe technique. Le code du modèle n'est pas disponible actuelle- ment, mais le sera au cours des prochaines semaines, lorsqu'il aura été adéquatement annoté et nettoyé. Dans l'intervalle, les personnes qui souhaitent accéder au code du modèle doivent communiquer directement avec David Fisman (david.fisman@utoronto.ca).

Remerciements : Les auteurs remercient Gabrielle Brankston, Shannon French, Tanya Rossi et Matthew Van Camp du Département de médecine des populations, Université de Guelph, pour leur aide à la compilation des données démographiques et des maladies chroniques. Les auteurs sont reconnaissants de l'aide et des commentaires reçus de Nelson Lee (Université de l'Alberta), Allison McGeer (Hôpital du Mont-Sinaï), Janine McCready (Hôpital Michael Garron, Toronto), Dick Zoutman (Réseau hospitalier de Scarborough), Jacqueline Willmore (Santé publique, Ottawa), Lydia Cheng (Santé publique, Peel), Monali Varia (Santé publique, Peel), Kristen Wheeler (Santé publique, Halton), Herveen Sachdeva (Santé publique, Toronto), Michael Finkelstein (Santé publique, Toronto), Monir Taha (Santé publique, Ottawa), Vera Etches (Santé publique, Ottawa), Isaac Bogoch (Réseau universitaire de santé), Chris Kandel (Réseau universitaire de santé et Hôpital Michael Garron), Jeff Powis (Hôpital Michael Garron) et Bart Harvey (Santé publique, Hamilton) pour la préparation des scénarios d'interventions plausibles testés dans cette analyse.

Accepté : Le 2 avril 2020

Auteur de correspondance : Ashleigh Tuite, ashleigh.tuite@utoronto.ca 